\title{
ON PROPERTIES OF SOLUTIONS FOR A FUNCTIONAL EQUATION
}

\author{
ZEQING LIU — SHIN Min KANG
}

\begin{abstract}
This paper studies properties of solutions for a functional equation arising in dynamic programming of multistage decision processes. Using the Banach fixed point theorem and the Mann iterative methods, we prove the existence and uniqueness of solutions and convergence of sequences generated by the Mann iterative methods for the functional equation in the Banach spaces $B C(S)$ and $B(S)$ and the complete metric space $B B(S)$, and discuss behaviors of solutions for the functional equation in the complete metric space $B B(S)$. Four examples illustrating the results presented in this paper are also provided.
\end{abstract}

\section{Introduction}

In the past decades, the existence of solutions for some classes of functional equations arising in dynamic programming in the complete metric space $B B(S)$ has been discussed by several authors, see, for instance, [1]-[15] and the references quoted therein. Bellman [1], Bhakta and Choudhury [4], Liu and Kang [8], [9], Liu et al. [12] and Liu et al. [15] studied the existence of solutions for the functional equations

$$
f(x)=\inf _{y \in D} \max \{u(x, y), f(a(x, y))\}, \quad \text { for all } x \in S,
$$

2010 Mathematics Subject Classification. 49L20, 90C39.

Key words and phrases. Functional equation, dynamic programming, continuous bounded solutions, bounded solutions, solutions, Mann iterative methods, Banach fixed point theorem.

This research was supported by the Science Research Foundation of Educational Department of Liaoning Province (L2012380). 
and

$$
f(x)=\inf _{y \in D} \max \{u(x, y), v(x, u) f(a(x, y))\}, \quad \text { for all } x \in S
$$

in the complete metric space $B B(S)$. Liu et al. [12] and Liu and Ume [11] obtained the existence, uniqueness and iterative approximations of solutions for the functional equations

$$
\begin{array}{ll}
f(x)=\sup _{y \in D} \max \{u(x, y), f(a(x, y))\}, & \text { for all } x \in S, \\
f(x)=\operatorname{opt}_{y \in D} \operatorname{opt}\{u(x, y), f(a(x, y))\}, & \text { for all } x \in S,
\end{array}
$$

in the complete metric space $B B(S)$, where opt stands for the sup or inf. However, relatively little is known about the existence of continuous bounded solutions and bounded solutions for the functional equations (1.1)-(1.4).

The aim of this paper is to introduce and study a more general functional equation arising in dynamic programming of multistage decision processes

$$
\begin{aligned}
f(x)= & \lambda \sup _{y \in D} \operatorname{opt}\{p(x, y), u(x, y) f(a(x, y))\} \\
& +(1-\lambda) \inf _{y \in D} \operatorname{opt}\{q(x, y), v(x, y) f(b(x, y))\}, \quad \text { for all } x \in S,
\end{aligned}
$$

where $\lambda \in[0,1]$ is a constant, $x$ and $y$ stand for the state and decision vectors, respectively, $a$ and $b$ denote the transformations of the processes, and $f(x)$ is the optimal return function with initial state $x$. It is clear that the functional equation (1.5) includes the functional equations (1.1)-(1.4) as special cases. Based on the Banach fixed point theorem, the Mann iterative methods and some techniques in nonlinear analysis, we provide sufficient conditions which guarantee the existence, uniqueness and iterative approximations of continuous bounded solutions, bounded solutions and solutions for the functional equation (1.5) in the Banach spaces $B C(S)$ and $B(S)$ and the complete metric space $B B(S)$, respectively, and prove some error estimates between the sequences generated by the Mann iterative methods and these solutions. Furthermore, we deduce properties of solutions for the functional equation (1.5) in the complete metric space $B B(S)$. Four example illustrating the results presented in this paper are included. Our results extend, improve and unify the corresponding results in [4], $[6],[8],[9],[11],[12]$ and $[15]$.

\section{Preliminaries}

In this section we introduce some notation and preliminary lemmas.

Throughout this paper, $(X,\|\cdot\|)$ and $\left(Y,\|\cdot\|^{\prime}\right)$ denote real Banach spaces, $S \subset X$ and $D \subset Y$ stand for the state space and decision space, respectively, 
$\mathbb{N}$ is the set of all positive integers, $\mathbb{N}_{0}=\{0\} \cup \mathbb{N}, \mathbb{R}=(-\infty,+\infty), \mathbb{R}^{+}=[0,+\infty)$ and $\mathbb{R}^{-}=(-\infty, 0]$. Define

$\Phi_{1}=\left\{\varphi: \varphi: \mathbb{R}^{+} \rightarrow \mathbb{R}^{+}\right.$is nondecreasing $\}$,

$\Phi_{2}=\left\{(\varphi, \psi): \varphi, \psi \in \Phi_{1}, \sum_{n=0}^{\infty} \psi\left(\varphi^{n}(t)\right)<+\infty\right.$ and $\psi(t)>0$ for all $\left.t>0\right\}$,

$B(S)=\{h: h: S \rightarrow \mathbb{R}$ is bounded $\}$,

$B C(S)=\{h: h \in B(S)$ is continuous $\}$,

$B B(S)=\{h: h: S \rightarrow \mathbb{R}$ is bounded on each bounded subsets of $S\}$.

Clearly, $\left(B(S),\|\cdot\|_{1}\right)$ and $\left(B C(S),\|\cdot\|_{1}\right)$ are Banach spaces with the norm $\|h\|_{1}=\sup _{x \in S}|h(x)|$. For each $k \in \mathbb{N}$ and $f, g \in B B(S)$, put

$$
d_{k}(h, g)=\sup \{|h(x)-g(x)|: x \in \bar{B}(0, k)\}, \quad d(h, g)=\sum_{k=1}^{\infty} \frac{1}{2^{k}} \cdot \frac{d_{k}(h, g)}{1+d_{k}(h, g)},
$$

where $\bar{B}(0, k)=\{x: x \in S$ and $\|x\| \leq k\}$. Obviously, $\left\{d_{k}\right\}_{k \in \mathbb{N}}$ is a countable family of pseudometrics in $B B(S)$. A sequence $\left\{x_{k}\right\}_{k \in \mathbb{N}}$ in $B B(S)$ is said to converge to a point $x \in B B(S)$ if $d_{k}\left(x_{n}, x\right) \rightarrow 0$ as $n \rightarrow \infty$ and $\left\{x_{n}\right\}_{n \in \mathbb{N}}$ is a Cauchy sequence if $d_{k}\left(x_{n}, x_{m}\right) \rightarrow 0$ as $n, m \rightarrow \infty$ for each $k \in \mathbb{N}$. It is clear that $(B B(S), d)$ is a complete metric space.

Lemma 2.1 ([9]). Let $E$ be a set, $p$ and $q: E \rightarrow \mathbb{R}$ be mappings. If $\underset{y \in E}{\operatorname{opt}} p(y)$ and $\underset{y \in E}{\operatorname{opt}} q(y)$ are bounded, then

$$
\left|\operatorname{opt}_{y \in E} p(y)-\operatorname{opt}_{y \in E} q(y)\right| \leq \sup _{y \in E}|p(y)-q(y)|
$$

Lemma 2.2 ([11]). Let $\alpha, \beta, \gamma$ and $\delta$ be in $\mathbb{R}$. Then

$$
|\operatorname{opt}\{\alpha, \beta\}-\operatorname{opt}\{\gamma, \delta\}| \leq \max \{|\alpha-\gamma|,|\beta-\delta|\} .
$$

\section{Properties of solutions for the functional equation (1.5)}

Now we derive the existence, uniqueness and iterative approximations of continuous bounded solutions and bounded solutions for the functional equation (1.5) in the Banach spaces $B C(S)$ and $B(S)$, respectively.

Theorem 3.1. Let $\lambda \in[0,1]$ and $\alpha \in(0,1)$ be constants. Assume that $S$ is compact, $p, q, u, v: S \times D \rightarrow \mathbb{R}$ and $a, b: S \times D \rightarrow S$ satisfy that

(C1) $p$ and $q$ are bounded in $S \times D$;

(C2) $\sup _{(x, y) \in S \times D} \max \{|u(x, y)|,|v(x, y)|\} \leq \alpha$; 
(C3) for each $\left(x_{0}, g\right) \in S \times\{u, v, p, q, a, b\}$,

$$
\lim _{x \rightarrow x_{0}} g(x, y)=g\left(x_{0}, y\right) \quad \text { uniformly for } y \in D .
$$

Then the functional equation (1.5) possesses a unique continuous bounded solution $w \in B C(S)$ such that

(C4) for each $w_{0} \in B C(S)$, the Mann iterative sequence $\left\{w_{n}\right\}_{n \in \mathbb{N}_{0}}$ defined by

$$
\begin{array}{r}
w_{n+1}(x)=\left(1-\alpha_{n}\right) w_{n}(x)+\alpha_{n}\left(\lambda \sup _{y \in D} \operatorname{opt}\left\{p(x, y), u(x, y) w_{n}(a(x, y))\right\}\right. \\
\left.+(1-\lambda) \inf _{y \in D} \operatorname{opt}\left\{q(x, y), v(x, y) w_{n}(b(x, y))\right\}\right),
\end{array}
$$

for all $(x, n) \in S \times \mathbb{N}_{0}$ and

$$
\left\{\alpha_{n}\right\}_{n \in \mathbb{N}_{0}} \subset[0,1] \text { and } \sum_{n=0}^{\infty} \alpha_{n}=+\infty
$$

converges to $w$ and has the error estimate:

$$
\left\|w_{n+1}-w\right\|_{1} \leq e^{-(1-\alpha) \sum_{i=0}^{n} \alpha_{i}}\left\|w_{0}-w\right\|_{1}, \quad \text { for all } n \in \mathbb{N}_{0} .
$$

Proof. Define a mapping $H: B C(S) \rightarrow B C(S)$ by

$$
\begin{aligned}
H h(x)=\lambda \sup _{y \in D} \operatorname{opt}\{p(x, y) & , u(x, y) h(a(x, y))\} \\
& +(1-\lambda) \inf _{y \in D} \operatorname{opt}\{q(x, y), v(x, y) h(b(x, y))\},
\end{aligned}
$$

for all $(x, h) \in S \times B C(S)$.

Firstly, we show that $H$ is a self mapping in $B C(S)$. Let $\left(x_{0}, h\right) \in S \times B C(S)$ and $\varepsilon>0$. In light of (C1), (C3) and the compactness of $S$, we infer that there exist constants $M \geq 1, \delta>0$ and $\delta_{1}>0$ such that

$$
\begin{aligned}
& \sup _{(x, y) \in S \times D} \max \{|h(x)|,|p(x, y)|,|q(x, y)|\} \leq M \\
& \max \left\{\left|r(x, y)-r\left(x_{0}, y\right)\right|: r \in\right.\{u, v, p, q\}\}<\frac{\varepsilon}{2 M}, \\
& \text { for all }(x, y) \in S \times D \text { with }\left\|x-x_{0}\right\|<\delta ; \\
&\left|h\left(x_{1}\right)-h\left(x_{2}\right)\right|<\frac{\varepsilon}{2 M}, \quad \text { for all } x_{1}, x_{2} \in S \text { with }\left\|x_{1}-x_{2}\right\|<\delta_{1} ; \\
& \max \left\{\left\|t(x, y)-t\left(x_{0}, y\right)\right\|: t \in\{a, b\}\right\}<\delta_{1}, \\
& \\
& \text { for all }(x, y) \in S \times D \text { with }\left\|x-x_{0}\right\|<\delta .
\end{aligned}
$$


In terms of (C2), (3.4)-(3.8), Lemmas 2.1 and 2.2, we know that

$$
\begin{aligned}
& \left|H h(x)-H h\left(x_{0}\right)\right|=\mid \lambda \sup _{y \in D} \operatorname{opt}\{p(x, y), u(x, y) h(a(x, y))\} \\
& +(1-\lambda) \inf _{y \in D} \operatorname{opt}\{q(x, y), v(x, y) h(b(x, y))\} \\
& -\lambda \sup \operatorname{opt}\left\{p\left(x_{0}, y\right), u\left(x_{0}, y\right) h\left(a\left(x_{0}, y\right)\right)\right\} \\
& y \in D \\
& -(1-\lambda) \inf _{y \in D} \operatorname{opt}\left\{q\left(x_{0}, y\right), v\left(x_{0}, y\right) h\left(b\left(x_{0}, y\right)\right)\right\} \\
& \leq \lambda \sup _{y \in D}\left|\operatorname{opt}\{p(x, y), u(x, y) h(a(x, y))\}-\operatorname{opt}\left\{p\left(x_{0}, y\right), u\left(x_{0}, y\right) h\left(a\left(x_{0}, y\right)\right)\right\}\right| \\
& +(1-\lambda) \sup _{y \in D} \mid \operatorname{opt}\{q(x, y), v(x, y) h(b(x, y))\} \\
& \text { - opt }\left\{q\left(x_{0}, y\right), v\left(x_{0}, y\right) h\left(b\left(x_{0}, y\right)\right)\right\} \mid \\
& \leq \lambda \sup _{y \in D} \max \left\{\left|p(x, y)-p\left(x_{0}, y\right)\right|,\left|u(x, y) h(a(x, y))-u\left(x_{0}, y\right) h\left(a\left(x_{0}, y\right)\right)\right|\right\} \\
& +(1-\lambda) \sup _{y \in D} \max \left\{\left|q(x, y)-q\left(x_{0}, y\right)\right|,\right. \\
& \left.\left|v(x, y) h(b(x, y))-v\left(x_{0}, y\right) h\left(b\left(x_{0}, y\right)\right)\right|\right\} \\
& \leq \lambda \sup _{y \in D} \max \left\{\frac{\varepsilon}{2 M},|h(a(x, y))|\left|u(x, y)-u\left(x_{0}, y\right)\right|\right. \\
& \left.+\left|u\left(x_{0}, y\right)\right|\left|h(a(x, y))-h\left(a\left(x_{0}, y\right)\right)\right|\right\} \\
& +(1-\lambda) \sup _{y \in D} \max \left\{\frac{\varepsilon}{2 M},|h(b(x, y))|\left|v(x, y)-v\left(x_{0}, y\right)\right|\right. \\
& \left.+\left|v\left(x_{0}, y\right)\right|\left|h(b(x, y))-h\left(b\left(x_{0}, y\right)\right)\right|\right\} \\
& <\lambda \max \left\{\frac{\varepsilon}{2 M}, M \cdot \frac{\varepsilon}{2 M}+M \cdot \frac{\varepsilon}{2 M}\right\} \\
& +(1-\lambda) \max \left\{\frac{\varepsilon}{2 M}, M \cdot \frac{\varepsilon}{2 M}+M \cdot \frac{\varepsilon}{2 M}\right\}=\varepsilon,
\end{aligned}
$$

for all $x \in S$ with $\left\|x-x_{0}\right\|<\delta$ and

$$
\begin{aligned}
|H h(x)|= & \mid \lambda \sup _{y \in D} \operatorname{opt}\{p(x, y), u(x, y) h(a(x, y))\} \\
& +(1-\lambda) \inf _{y \in D} \operatorname{opt}\{q(x, y), v(x, y) h(b(x, y))\} \mid \\
\leq & \lambda \sup _{y \in D}|\operatorname{opt}\{p(x, y), u(x, y) h(a(x, y))\}| \\
& +(1-\lambda) \sup _{y \in D}|\operatorname{opt}\{q(x, y), v(x, y) h(b(x, y))\}|
\end{aligned}
$$




$$
\begin{aligned}
\leq & \lambda \sup _{y \in D} \max \{|p(x, y)|,|u(x, y)||h(a(x, y))|\} \\
& \quad+(1-\lambda) \sup _{y \in D} \max \{|q(x, y)|,|v(x, y)||h(b(x, y))|\} \\
\leq & \lambda \max \{M, \alpha M\}+(1-\lambda) \max \{M, \alpha M\}=M,
\end{aligned}
$$

for all $x \in S$, which yield that $H h$ is continuous and bounded in $S$. That is, $H$ maps $B C(S)$ into $B C(S)$.

Secondly, we show that $H$ is a contraction mapping in $B C(S)$. Notice that (3.4), Lemmas 2.1 and 2.2 ensure that

$$
\begin{aligned}
|H h(x)-H g(x)|= & \mid \lambda \sup _{y \in D} \operatorname{opt}\{p(x, y), u(x, y) h(a(x, y))\} \\
& +(1-\lambda) \inf _{y \in D} \operatorname{opt}\{q(x, y), v(x, y) h(b(x, y))\} \\
& -\lambda \sup _{y \in D} \operatorname{opt}\{p(x, y), u(x, y) g(a(x, y))\} \\
& -(1-\lambda) \inf _{y \in D} \operatorname{opt}\{q(x, y), v(x, y) g(b(x, y))\} \mid \\
\leq & \lambda \sup _{y \in D} \mid \operatorname{opt}\{p(x, y), u(x, y) h(a(x, y))\} \\
& -\operatorname{opt}\{p(x, y), u(x, y) g(a(x, y))\} \mid \\
& +(1-\lambda) \sup _{y \in D} \mid \operatorname{opt}\{q(x, y), v(x, y) h(b(x, y))\} \\
& -\operatorname{opt}\{q(x, y), v(x, y) g(b(x, y))\} \mid \\
\leq & \lambda \sup _{y \in D}\{|u(x, y) \| h(a(x, y))-g(a(x, y))|\} \\
& +(1-\lambda) \sup _{y \in D}\{|v(x, y) \| h(b(x, y))-g(b(x, y))|\} \\
\leq & \lambda \alpha\|h-g\|_{1}+(1-\lambda) \alpha\|h-g\|_{1}=\alpha\|h-g\|_{1},
\end{aligned}
$$

for all $x \in S, h, g \in B C(S)$, which means that

$$
\|H h-H g\|_{1} \leq \alpha\|h-g\|_{1}, \quad \text { for all } h, g \in B C(S),
$$

that is, $H$ is a contraction mapping in $B C(S)$. Thus the Banach fixed point theorem guarantees that $H$ has a unique fixed point $w \in B C(S)$, which is a unique continuous bounded solution of the functional equation (1.5) in $B C(S)$.

Thirdly, we show (C4). Note that, for all $x \in S$,

$$
\begin{aligned}
w(x)=\lambda \sup _{y \in D} \operatorname{opt}\{p(x, y) & , u(x, y) w(a(x, y))\} \\
& +(1-\lambda) \inf _{y \in D} \operatorname{opt}\{q(x, y), v(x, y) w(b(x, y))\} .
\end{aligned}
$$

By virtue of (3.1), (3.4), (3,9) and (3.10), we derive that

$$
\left|w_{n+1}(x)-w(x)\right|=\mid\left(1-\alpha_{n}\right) w_{n}(x)+\alpha_{n}\left(\lambda \sup _{y \in D} \operatorname{opt}\left\{p(x, y), u(x, y) w_{n}(a(x, y))\right\}\right.
$$




$$
\begin{aligned}
& \left.+(1-\lambda) \inf _{y \in D} \operatorname{opt}\left\{q(x, y), v(x, y) w_{n}(b(x, y))\right\}\right)-w(x) \mid \\
\leq & \left(1-\alpha_{n}\right)\left|w_{n}(x)-w(x)\right|+\alpha_{n} \mid \lambda \sup _{y \in D} \operatorname{opt}\left\{p(x, y), u(x, y) w_{n}(a(x, y))\right\} \\
& +(1-\lambda) \inf _{y \in D} \operatorname{opt}\left\{q(x, y), v(x, y) w_{n}(b(x, y))\right\} \\
& -\lambda \sup _{y \in D} \operatorname{opt}\{p(x, y), u(x, y) w(a(x, y))\} \\
& -(1-\lambda) \inf _{y \in D} \operatorname{opt}\{q(x, y), v(x, y) w(b(x, y))\} \mid \\
\leq & \left(1-\alpha_{n}\right)\left|w_{n}(x)-w(x)\right|+\alpha_{n}\left|H w_{n}(x)-H w(x)\right| \\
\leq & \left(1-\alpha_{n}\right)\left\|w_{n}-w\right\|_{1}+\alpha_{n} \alpha\left|w_{n}(x)-w(x)\right| \leq\left(1-(1-\alpha) \alpha_{n}\right)\left\|w_{n}-w\right\|_{1},
\end{aligned}
$$

for all $(x, n) \in S \times \mathbb{N}_{0}$, which implies that

$$
\begin{aligned}
\left\|w_{n+1}-w\right\|_{1} & \leq\left(1-(1-\alpha) \alpha_{n}\right)\left\|w_{n}-w\right\|_{1} \\
& \leq e^{-(1-\alpha) \alpha_{n}}\left\|w_{n}-w\right\|_{1} \leq e^{-(1-\alpha) \sum_{i=0}^{n} \alpha_{i}}\left\|w_{0}-w\right\|_{1},
\end{aligned}
$$

for all $n \in \mathbb{N}_{0}$, which together with (3.3) gives that the Mann iterative sequence $\left\{w_{n}\right\}_{n \in \mathbb{N}_{0}}$ converges to $w$. This completes the proof.

As in the proof of Theorem 3.1, we infer immediately the following result.

Theorem 3.2. Let $\lambda \in[0,1]$ and $\alpha \in(0,1)$ be constants. Assume that $p, q, u, v: S \times D \rightarrow \mathbb{R}$ and $a, b: S \times D \rightarrow S$ satisfy (C1) and (C2). Then the functional equation (1.5) possesses a unique bounded solution $w \in B(S)$ and for each $w_{0} \in B(S)$, the Mann iterative sequence $\left\{w_{n}\right\}_{n \in \mathbb{N}_{0}}$ defined by (3.1) and (3.2) converges to $w$ and satisfies (C4).

Now we give two examples as applications of Theorems 3.1 and 3.2.

EXAMPLE 3.3. Consider the functional equation

$$
\begin{aligned}
f(x)= & \lambda \sup _{y \in \mathbb{R}^{+}} \text {opt }\left\{\frac{x^{4}}{x+y^{3}}, \frac{\cos \left(x^{2} y^{3}\right)}{y^{4}+3} f\left(\frac{x^{4}+y^{2}}{x^{3}+y^{2}}\right)\right\} \\
& +(1-\lambda) \inf _{y \in \mathbb{R}^{+}} \text {opt }\left\{\frac{x^{6}}{x^{2}+y^{2}}, \frac{x^{6} \sin \left(x^{5} y^{2}\right)}{2 x^{6}+y^{3}} f\left(\frac{x^{2}+15 x+y^{2}}{x^{2}+y^{2}}\right)\right\},
\end{aligned}
$$

for all $x \in[1,20]$.

Let $X=Y=\mathbb{R}, S=[1,20], D=\mathbb{R}^{+}, \alpha=5 / 6, \lambda \in[0,1], p, q, u, v: S \times D \rightarrow \mathbb{R}$ and $a, b: S \times D \rightarrow S$ be defined by

$$
\begin{array}{ll}
p(x, y)=\frac{x^{4}}{x+y^{3}}, & q(x, y)=\frac{x^{6}}{x^{2}+y^{2}} \\
u(x, y)=\frac{\cos \left(x^{2} y^{3}\right)}{y^{4}+3}, & v(x, y)=\frac{x^{6} \sin \left(x^{5} y^{2}\right)}{2 x^{6}+y^{3}}, \\
a(x, y)=\frac{x^{4}+y^{2}}{x^{3}+y^{2}}, & b(x, y)=\frac{x^{2}+15 x+y^{2}}{x^{2}+y^{2}}
\end{array}
$$


for all $(x, y) \in S \times D$. Clearly, the conditions of Theorem 3.1 hold. It follows from Theorem 3.1 that the functional equation (3.11) has a unique continuous bounded solution $w \in B C(S)$, and for each $w_{0} \in B C(S)$, the Mann iterative sequence $\left\{w_{n}\right\}_{n \in \mathbb{N}_{0}}$ defined by

$$
\begin{aligned}
w_{n+1}(x)= & \left(1-\frac{1}{\sqrt{5 n+6+(-1)^{n} \sin n}}\right) w_{n}(x)+\frac{1}{\sqrt{5 n+6+(-1)^{n} \sin n}} \\
& \times\left(\lambda \sup _{y \in \mathbb{R}^{+}} \operatorname{opt}\left\{\frac{x^{4}}{x+y^{3}}, \frac{\cos \left(x^{2} y^{3}\right)}{y^{4}+3} w_{n}\left(\frac{x^{4}+y^{2}}{x^{3}+y^{2}}\right)\right\}\right. \\
& \left.+(1-\lambda) \inf _{y \in \mathbb{R}^{+}} \operatorname{opt}\left\{\frac{x^{6}}{x^{2}+y^{2}}, \frac{x^{6} \sin \left(x^{5} y^{2}\right)}{2 x^{6}+y^{3}} w_{n}\left(\frac{x^{2}+15 x+y^{2}}{x^{2}+y^{2}}\right)\right\}\right),
\end{aligned}
$$

for all $(x, n) \in[1,20] \times \mathbb{N}_{0}$ and $\alpha_{n}=1 / \sqrt{5 n+6+(-1)^{n} \sin n}$, for all $n \in \mathbb{N}_{0}$ converges to $w$ and has the error estimate:

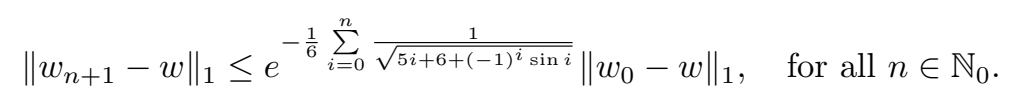

Example 3.4. Consider the functional equation

$$
\begin{aligned}
f(x)= & \lambda \sup _{y \in \mathbb{R}^{-}} \text {opt }\left\{\frac{x^{7} y^{4}+\ln \left(1+\frac{1}{1+x^{3} y^{8}}\right)}{x^{7} y^{4}+1}, \frac{x y^{2}+1}{x^{2}+y^{4}+2} f\left(\frac{x^{5}+x^{3} y^{8}+y^{2}}{x^{2} y^{2}+1}\right)\right\} \\
& +(1-\lambda) \inf _{y \in \mathbb{R}^{-}} \text {opt }\left\{\frac{x^{3}+y}{(x+1)^{3}+y^{2}}, \frac{x^{3} y^{2} \cos ^{5}\left(x^{2} y^{9}\right)}{4 x^{3} y^{2}+1} f\left(\frac{x^{3} y^{4}}{x^{4}|y|+1}\right)\right\}
\end{aligned}
$$

for all $x \in \mathbb{R}^{+}$. Let $X=Y=\mathbb{R}, S=\mathbb{R}^{+}, D=\mathbb{R}^{-}, \alpha=3 / 4, \lambda \in[0,1]$, $p, q, u, v: S \times D \rightarrow \mathbb{R}$ and $a, b: S \times D \rightarrow S$ be defined by

$$
\begin{aligned}
p(x, y) & =\frac{x^{7} y^{4}+\ln \left(1+\frac{1}{1+x^{3} y^{8}}\right)}{x^{7} y^{4}+1}, & q(x, y) & =\frac{x^{3}+y}{(x+1)^{3}+y^{2}}, \\
u(x, y) & =\frac{x y^{2}+1}{x^{2}+y^{4}+2}, & v(x, y) & =\frac{x^{3} y^{2} \cos ^{5}\left(x^{2} y^{9}\right)}{4 x^{3} y^{2}+1}, \\
a(x, y) & =\frac{x^{5}+x^{3} y^{8}+y^{2}}{x^{2} y^{2}+1}, & b(x, y) & =\frac{x^{3} y^{4}}{x^{4}|y|+1},
\end{aligned}
$$

for all $(x, y) \in S \times D$. Obviously, the conditions of Theorem 3.2 hold. Thus Theorem 3.2 means that the functional equation (3.12) has a unique bounded solution $w \in B(S)$, and for any $w_{0} \in B C(S)$, the Mann iterative sequence $\left\{w_{n}\right\}_{n \in \mathbb{N}_{0}}$ defined by

$$
\begin{gathered}
w_{n+1}(x)=\left(1-\frac{n^{2}+3 n+1}{n^{3}+4 n^{2}-3 n+4+\ln (n+3)}\right) w_{n}(x) \\
+\frac{n^{2}+3 n+1}{n^{3}+4 n^{2}-3 n+4+\ln (n+3)}
\end{gathered}
$$




$$
\begin{aligned}
& \times\left(\lambda \sup _{y \in \mathbb{R}^{-}} \text {opt }\left\{\frac{x^{7} y^{4}+\ln \left(1+\frac{1}{1+x^{3} y^{8}}\right)}{x^{7} y^{4}+1}, \frac{x y^{2}+1}{x^{2}+y^{4}+2} w_{n}\left(\frac{x^{5}+x^{3} y^{8}+y^{2}}{x^{2} y^{2}+1}\right)\right\}\right. \\
& \left.+(1-\lambda) \inf _{y \in \mathbb{R}^{-}} \operatorname{opt}\left\{\frac{x^{3}+y}{(x+1)^{3}+y^{2}}, \frac{x^{3} y^{2} \cos ^{5}\left(x^{2} y^{9}\right)}{4 x^{3} y^{2}+1} w_{n}\left(\frac{x^{3} y^{4}}{x^{4}|y|+1}\right)\right\}\right),
\end{aligned}
$$

for all $(x, n) \in \mathbb{R}^{+} \times \mathbb{N}_{0}$ and

$$
\alpha_{n}=\frac{n^{2}+3 n+1}{n^{3}+4 n^{2}-3 n+4+\ln (n+3)}, \quad \text { for all } n \in \mathbb{N}_{0}
$$

converges to $w$ and has the error estimate:

$$
\left\|w_{n+1}-w\right\|_{1} \leq e^{-\frac{1}{4} \sum_{i=0}^{n} \frac{i^{2}+3 i+1}{i^{3}+4 i^{2}-3 i+4+\ln (i+3)}}\left\|w_{0}-w\right\|_{1}, \quad \text { for all } n \in \mathbb{N}_{0} .
$$

Next we study the behaviors of solutions and iterative approximations for the functional equation (1.5) in the complete metric space $B B(S)$.

Theorem 3.5. Let $\lambda \in[0,1]$ and $\alpha \in(0,1)$ be constants and (3.2) hold. Assume that $p, q, u, v: S \times D \rightarrow \mathbb{R}$ and $a, b: S \times D \rightarrow S$ satisfy that

(C5) $p$ and $q$ are bounded on $\bar{B}(0, k) \times D$, for all $k \in \mathbb{N}$;

(C6) $\sup _{\max }\{|u(x, y)|,|v(x, y)|\} \leq \alpha$, for all $k \in \mathbb{N}$;

(C7) $\sup _{(x, y) \in \bar{B}(0, k) \times D} \max \{\|a(x, y)\|,\|b(x, y)\|\} \leq k$, for all $k \in \mathbb{N}$.

Then the functional equation (1.5) possesses a unique solution $w \in B B(S)$ such that

(C8) for each $w_{0} \in B B(S)$, the Mann iterative sequence $\left\{w_{n}\right\}_{n \in \mathbb{N}_{0}}$ defined by

$$
\begin{array}{r}
w_{n+1}(x)=\left(1-\alpha_{n}\right) w_{n}(x)+\alpha_{n}\left(\lambda \sup _{y \in D} \operatorname{opt}\left\{p(x, y), u(x, y) w_{n}(a(x, y))\right\}\right. \\
\left.+(1-\lambda) \inf _{y \in D} \operatorname{opt}\left\{q(x, y), v(x, y) w_{n}(b(x, y))\right\}\right),
\end{array}
$$

for all $(x, k, n) \in \bar{B}(0, k) \times \mathbb{N} \times \mathbb{N}_{0}$, converges to $w$ and has the error estimate:

$$
d_{k}\left(w_{n+1}, w\right) \leq e^{-(1-\alpha) \sum_{i=0}^{n} \alpha_{i}} d_{k}\left(w_{0}, w\right), \quad \text { for all }(k, n) \in \mathbb{N} \times \mathbb{N}_{0} .
$$

Proof. Define a mapping $H: B B(S) \rightarrow B B(S)$ by

$$
\begin{aligned}
H h(x)=\lambda \sup _{y \in D} \operatorname{opt}\{p(x, y) & , u(x, y) h(a(x, y))\} \\
& +(1-\lambda) \inf _{y \in D} \operatorname{opt}\{q(x, y), v(x, y) h(b(x, y))\},
\end{aligned}
$$


for all $(x, k, h) \in \bar{B}(0, k) \times \mathbb{N} \times B B(S)$. Put $(k, h) \in \mathbb{N} \times B B(S)$. It follows from $(\mathrm{C} 5)$ and $(\mathrm{C} 7)$ that there exists $\gamma>0$ such that

$$
\sup _{(x, y) \in \bar{B}(0, k) \times D} \max \{|p(x, y)|,|q(x, y)|, \mid h(a(x, y)|,| h(b(x, y)) \mid\} \leq \gamma,
$$

which together with $(\mathrm{C} 6),(3.15)$ and Lemma 2.1 yields that

$$
\begin{aligned}
|H h(x)|= & \mid \lambda \operatorname{opt}_{y \in D} \sup \{p(x, y), u(x, y) h(a(x, y))\} \\
& +(1-\lambda) \inf _{y \in D} \operatorname{opt}\{q(x, y), v(x, y) h(b(x, y))\} \mid \\
\leq & \lambda \sup _{y \in D} \max \{|p(x, y)|,|u(x, y)||h(a(x, y))|\} \\
& +(1-\lambda) \sup _{y \in D} \max \{|q(x, y)|,|v(x, y)||h(b(x, y))|\} \\
\leq & \lambda \max \{\gamma, \alpha \gamma\}+(1-\lambda) \max \{\gamma, \alpha \gamma\}=\gamma,
\end{aligned}
$$

for all $(x, k, h) \in \bar{B}(0, k) \times \mathbb{N} \times B B(S)$, which means that $H$ is a self mapping in $B B(S)$.

On account of (3.15), (C6), (C7), Lemmas 2.1 and 2.2, we derive that

$$
\begin{aligned}
\mid H h(x) & -H g(x)|=| \lambda \sup _{y \in D} \operatorname{opt}\{p(x, y), u(x, y) h(a(x, y))\} \\
& +(1-\lambda) \inf _{y \in D} \operatorname{opt}\{q(x, y), v(x, y) h(b(x, y))\} \\
& -\lambda \sup _{y \in D} \operatorname{opt}\{p(x, y), u(x, y) g(a(x, y))\} \\
& -(1-\lambda) \inf _{y \in D} \operatorname{opt}\{q(x, y), v(x, y) g(b(x, y))\} \mid \\
\leq & \lambda \sup _{y \in D}|\operatorname{opt}\{p(x, y), u(x, y) h(a(x, y))\}-\operatorname{opt}\{p(x, y), u(x, y) g(a(x, y))\}| \\
& +(1-\lambda) \sup _{y \in D} \mid \operatorname{opt}\{q(x, y), v(x, y) h(b(x, y))\} \\
& -\operatorname{opt}\{q(x, y), v(x, y) g(b(x, y))\} \mid \\
\leq & \lambda \sup _{y \in D}\{|u(x, y)||h(a(x, y))-g(a(x, y))|\} \\
& +(1-\lambda) \sup _{y \in D}\{|v(x, y)||h(b(x, y))-g(b(x, y))|\} \\
\leq & \lambda \alpha d_{k}(h, g)+(1-\lambda) \alpha d_{k}(h, g)=\alpha d_{k}(h, g),
\end{aligned}
$$

for all $(x, k, h, g) \in \bar{B}(0, k) \times \mathbb{N} \times B B(S) \times B B(S)$, which yields that

$$
d_{k}(H h, H g) \leq \alpha d_{k}(h, g), \quad \text { for all }(k, h, g) \in \mathbb{N} \times B B(S) \times B B(S) .
$$


For $z_{0}=0 \in B B(S)$, put

$$
\begin{aligned}
z_{n}(x)=\lambda \sup _{y \in D} \operatorname{opt}\left\{p(x, y), u(x, y) z_{n-1}(a(x, y))\right\} & \\
& +(1-\lambda) \inf _{y \in D} \operatorname{opt}\left\{q(x, y), v(x, y) z_{n-1}(b(x, y))\right\},
\end{aligned}
$$

for all $(x, n) \in S \times \mathbb{N}$. In view of (3.15), (3.16) and (3.17), we obtain that

$$
\begin{aligned}
d_{k}\left(z_{n}, z_{n+m}\right) & \leq \sum_{i=n}^{n+m-1} d_{k}\left(z_{i}, z_{i+1}\right)=\sum_{i=n}^{n+m-1} d_{k}\left(H z_{i-1}, H z_{i}\right) \\
& \leq \sum_{i=n}^{n+m-1} \alpha d_{k}\left(z_{i-1}, z_{i}\right) \leq \sum_{i=n}^{n+m-1} \alpha^{i} d_{k}\left(z_{0}, z_{1}\right) \leq \frac{\alpha^{n}}{1-\alpha} d_{k}\left(z_{0}, z_{1}\right),
\end{aligned}
$$

for all $(k, n, m) \in \mathbb{N} \times \mathbb{N}_{0} \times \mathbb{N}$, which implies that $\left\{z_{n}\right\}_{n \in \mathbb{N}_{0}}$ is a Cauchy sequence in $B B(S)$. Consequently, there exists some $w \in B B(S)$ such that $\left\{z_{n}\right\}_{n \in \mathbb{N}_{0}}$ converges to $w$. Notice that (3.16) ensures that

$$
d_{k}(w, H w) \leq d_{k}\left(w, z_{n+1}\right)+d_{k}\left(H z_{n}, H w\right) \leq d_{k}\left(w, z_{n+1}\right)+\alpha d_{k}\left(z_{n}, w\right) \rightarrow 0,
$$

for all $k \in \mathbb{N}$ as $n \rightarrow \infty$, that is, $d_{k}(w, H w)=0$, for all $k \in \mathbb{N}$, which yields that

$$
d(w, H w)=\sum_{k=1}^{\infty} \frac{1}{2^{k}} \cdot \frac{d_{k}(w, H w)}{1+d_{k}(w, H w)}=0,
$$

that is, $w=H w$.

Suppose that there exists $t \in B B(S) \backslash\{w\}$ with $t=H t$. It follows that $d_{k_{0}}(w, t)>0$ for some $k_{0} \in \mathbb{N}$. In terms of $(3.16)$, we see that

$$
0<d_{k_{0}}(w, t)=d_{k_{0}}(H w, H t) \leq \alpha d_{k_{0}}(w, t)<d_{k_{0}}(w, t)
$$

which is impossible. Hence the mapping $H$ has a unique fixed point $w \in B B(S)$, which is a unique solution of the functional equation (1.5) in $B B(S)$.

Finally, we show that (C8) holds. Using (3.13), (3.15), (3.16) and Lemma 2.1, we infer that

$$
\begin{aligned}
\left|w_{n+1}(x)-w(x)\right|= & \mid\left(1-\alpha_{n}\right) w_{n}(x)+\alpha_{n}\left(\lambda \sup _{y \in D} \operatorname{opt}\left\{p(x, y), u(x, y) w_{n}(a(x, y))\right\}\right. \\
& \left.+(1-\lambda) \inf _{y \in D} \operatorname{opt}\left\{q(x, y), v(x, y) w_{n}(b(x, y))\right\}\right)-w(x) \mid \\
\leq & \left(1-\alpha_{n}\right)\left|w_{n}(x)-w(x)\right| \\
& +\alpha_{n} \mid \lambda \sup _{y \in D} \operatorname{opt}\left\{p(x, y), u(x, y) w_{n}(a(x, y))\right\} \\
& +(1-\lambda) \inf _{y \in D} \operatorname{opt}\left\{q(x, y), v(x, y) w_{n}(b(x, y))\right\} \\
& -\lambda \sup _{y \in D} \operatorname{opt}\{p(x, y), u(x, y) w(a(x, y))\}
\end{aligned}
$$




$$
\begin{aligned}
& -(1-\lambda) \inf _{y \in D} \operatorname{opt}\{q(x, y), v(x, y) w(b(x, y))\} \\
\leq & \left(1-\alpha_{n}\right) d_{k}\left(w_{n}, w\right)+\alpha_{n} d_{k}\left(H w_{n}, H w\right) \\
\leq & \left(1-(1-\alpha) \alpha_{n}\right) d_{k}\left(w_{n}, w\right),
\end{aligned}
$$

for all $(x, k, n) \in \bar{B}(0, k) \times \mathbb{N} \times \mathbb{N}_{0}$, which yields that

$$
d_{k}\left(w_{n+1}, w\right) \leq e^{-(1-\alpha) \alpha_{n}} d_{k}\left(w_{n}, w\right) \leq e^{-(1-\alpha) \sum_{i=0}^{n} \alpha_{i}} d_{k}\left(w_{0}, w\right),
$$

for all $(k, n) \in \mathbb{N} \times \mathbb{N}_{0}$, which together with (3.2) yields that $\left\{w_{n}\right\}_{n \in \mathbb{N}_{0}}$ converges to $w$. This completes the proof.

REMARK 3.6. Theorem 3.5 extends and improves Theorems 3.4 and 3.5 in [5]. The example below shows that Theorem 3.5 extends substantially Theorems 3.4 and 3.5 in [5].

EXAMPLE 3.7. Consider the functional equation

$$
\begin{aligned}
& f(x)=\lambda \sup _{y \in \mathbb{R}} \text { opt }\left\{\frac{x^{17} y^{6} \sin ^{9}\left(x^{5} y^{7}\right)}{x^{4} y^{6}+2}, \frac{\cos ^{5}\left(x^{4} y^{3}\right)}{2+\ln \left(x^{2} y^{2}+2\right)} f\left(\frac{x^{9} y^{2} \sin \left(x^{5} y^{4}\right)}{x^{8} y^{2}+1}\right)\right\} \\
& +(1-\lambda) \inf _{y \in \mathbb{R}} \text { opt }\left\{\frac{x^{51}-\sqrt{x^{42}+1}}{\left(x^{2}-y^{3}\right)^{2}+1}, \frac{\sin \left(x^{2} y\right)}{3+\cos ^{7}\left(x^{4} y^{9}\right)} f\left(\frac{x^{2} y^{3} \cos \left(x^{3} y^{6}\right)}{\left|x y^{3}\right|+1}\right)\right\}
\end{aligned}
$$

for all $x \in \mathbb{R}$. Put $X=Y=S=D=\mathbb{R}, \lambda \in[0,1]$ and $\alpha=2 /(2+\ln 2)$. Let $p, q, u, v: S \times D \rightarrow \mathbb{R}$ and $a, b: S \times D \rightarrow S$ be defined by

$$
\begin{aligned}
p(x, y) & =\frac{x^{17} y^{6} \sin ^{9}\left(x^{5} y^{7}\right)}{x^{4} y^{6}+2}, & q(x, y) & =\frac{x^{51}-\sqrt{x^{42}+1}}{\left(x^{2}-y^{3}\right)^{2}+1}, \\
u(x, y) & =\frac{\cos ^{5}\left(x^{4} y^{3}\right)}{2+\ln \left(x^{2} y^{2}+2\right)}, & v(x, y) & =\frac{\sin \left(x^{2} y\right)}{3+\cos ^{7}\left(x^{4} y^{9}\right)}, \\
a(x, y) & =\frac{x^{9} y^{2} \sin \left(x^{5} y^{4}\right)}{x^{8} y^{2}+1}, & b(x, y) & =\frac{x^{2} y^{3} \cos \left(x^{3} y^{6}\right)}{\left|x y^{3}\right|+1},
\end{aligned}
$$

for all $(x, y) \in S \times D$. It is clear that the conditions of Theorem 3.5 hold. It follows from Theorem 3.5 that the functional equation (3.18) possesses a unique solution $w \in B B(S)$, and for each $w_{0} \in B B(S)$, the Mann iterative sequence $\left\{w_{n}\right\}_{n \in \mathbb{N}_{0}}$ defined by

$$
\begin{aligned}
& w_{n+1}(x)=\frac{\sqrt{4 n+2}+3+(-1)^{n}}{\sqrt{n^{2}+1}+\sqrt{4 n+2}+5} w_{n}(x)+\frac{\sqrt{n^{2}+1}+2-(-1)^{n}}{\sqrt{n^{2}+1}+\sqrt{4 n+2}+5} \\
& \times\left(\lambda \sup _{y \in D} \operatorname{opt}\left\{\frac{x^{17} y^{6} \sin ^{9}\left(x^{5} y^{7}\right)}{x^{4} y^{6}+2}, \frac{\cos ^{5}\left(x^{4} y^{3}\right)}{2+\ln \left(x^{2} y^{2}+2\right)} w_{n}\left(\frac{x^{9} y^{2} \sin \left(x^{5} y^{4}\right)}{x^{8} y^{2}+1}\right)\right\}\right. \\
& \left.+(1-\lambda) \inf _{y \in D} \operatorname{opt}\left\{\frac{x^{51}-\sqrt{x^{42}+1}}{\left(x^{2}-y^{3}\right)^{2}+1}, \frac{\sin \left(x^{2} y\right)}{3+\cos ^{7}\left(x^{4} y^{9}\right)} w_{n}\left(\frac{x^{2} y^{3} \cos \left(x^{3} y^{6}\right)}{\left|x y^{3}\right|+1}\right)\right\}\right),
\end{aligned}
$$


for all $(x, k, n) \in \bar{B}(0, k) \times \mathbb{N} \times \mathbb{N}_{0}$ and

$$
\alpha_{n}=\frac{\sqrt{n^{2}+1}+2-(-1)^{n}}{\sqrt{n^{2}+1}+\sqrt{4 n+2}+5}, \quad \text { for all } n \in \mathbb{N}_{0}
$$

converges to $w$ and has the error estimate:

$$
d_{k}\left(w_{n+1}, w\right) \leq e^{-\frac{\ln 2}{2+\ln 2} \sum_{i=0}^{n} \frac{\sqrt{i^{2}+1}+2-(-1)^{i}}{\sqrt{i^{2}+1}+\sqrt{4 i+2}+5}} d_{k}\left(w_{0}, w\right), \quad \text { for all }(k, n) \in \mathbb{N} \times \mathbb{N}_{0} .
$$

But Theorems 3.4, 3.5 in [5] are unapplicable for the functional equation (3.18).

Theorem 3.8. Let $\lambda \in[0,1]$ and $\beta \in(0,1]$ be constants and $(\varphi, \psi) \in \Phi_{2}$. Assume that $p, q, u, v: S \times D \rightarrow \mathbb{R}, a, b: S \times D \rightarrow S$ and $c: S \rightarrow S$ satisfy that:

(C9) $\sup _{(x, y) \in \bar{B}(0, k) \times D} \max \{|p(x, y)|,|q(x, y)|\} \leq \psi(\|x\|)$, for all $k \in \mathbb{N}$;

(C10) $\sup _{(x, y) \in \bar{B}(0, k) \times D} \max \{|u(x, y)|,|v(x, y)|\} \leq 1$, for all $k \in \mathbb{N}$;

$(\mathrm{C} 11) \max \left\{\sup _{x \in \bar{B}(0, k)}\|c(x)\|, \sup _{(x, y) \in \bar{B}(0, k) \times D} \max \{\|a(x, y)\|,\|b(x, y)\|\}\right\} \leq \varphi(\|x\|)$, for all $k \in \mathbb{N}$.

Then the functional equation (1.5) possesses a solution $w \in B B(S)$ such that

(C12) for each $w_{0} \in B B(S)$ with $\left|w_{0}(x)\right| \leq \psi(\|x\|)$, for all $(x, k) \in \bar{B}(0, k) \times \mathbb{N}$, the iterative sequence $\left\{w_{n}\right\}_{n \in \mathbb{N}_{0}}$ defined by

$$
\begin{aligned}
w_{n+1}(x)= & (1-\beta) w_{n}(c(x)) \\
& +\beta\left(\lambda \sup _{y \in D} \operatorname{opt}\left\{p(x, y), u(x, y) w_{n}(a(x, y))\right\}\right. \\
& \left.\quad+(1-\lambda) \inf _{y \in D} \operatorname{opt}\left\{q(x, y), v(x, y) w_{n}(b(x, y))\right\}\right),
\end{aligned}
$$

for all $(x, k, n) \in \bar{B}(0, k) \times \mathbb{N} \times \mathbb{N}_{0}$, converges to $w$ and

$$
d_{k}\left(w_{n}, w\right) \leq \sum_{j=n-1}^{\infty} \psi\left(\varphi^{j}(k)\right), \quad \text { for all }(k, n) \in \mathbb{N} \times \mathbb{N}
$$

(C13) $|w(x)| \leq \sum_{n=0}^{\infty} \psi\left(\varphi^{n}(\|x\|)\right)$, for all $(x, k) \in \bar{B}(0, k) \times \mathbb{N}$;

(C14) $\lim _{n \rightarrow \infty} w\left(x_{n}\right)=0$, for all $\left(x_{0}, k\right) \in \bar{B}(0, k) \times \mathbb{N},\left\{y_{n}\right\}_{n \in \mathbb{N}} \subset D$ with $x_{n} \in$ $\left\{c\left(x_{n-1}\right), a\left(x_{n-1}, y_{n}\right), b\left(x_{n-1}, y_{n}\right)\right\}$, for all $n \in \mathbb{N}$;

(C15) $w$ is a unique solution of the functional equation (1.5) relative to $(\mathrm{C} 14)$.

Proof. Now we show that

$$
\varphi(t)<t, \quad \text { for all } t>0 \text {. }
$$


Suppose that there exists some $t_{0}>0$ such that $\varphi\left(t_{0}\right) \geq t_{0}>0$. Observe that $\sum_{n=0}^{\infty} \psi\left(\varphi^{n}\left(t_{0}\right)\right)<+\infty$ and $(\varphi, \psi) \in \Phi_{2}$. It follows that

$0<\psi\left(t_{0}\right) \leq \psi\left(\varphi\left(t_{0}\right)\right) \leq \ldots \leq \psi\left(\varphi^{n-1}\left(t_{0}\right)\right) \leq \psi\left(\varphi^{n}\left(t_{0}\right)\right), \quad$ for all $n \in \mathbb{N}$,

which implies that $\lim _{n \rightarrow \infty} \psi\left(\varphi^{n}\left(t_{0}\right)\right) \geq \psi\left(t_{0}\right)>0$, which yields that $\sum_{n=0}^{\infty} \psi\left(\varphi^{n}\left(t_{0}\right)\right)$ $=+\infty$, which is absurd. Hence (3.20) holds.

Define a mapping $H: B B(S) \rightarrow B B(S)$ by

(3.21) $H h(x)=\lambda A h(x)+(1-\lambda) B h(x), \quad$ for all $(x, k, h) \in \bar{B}(0, k) \times \mathbb{N} \times B B(S)$,

where, for all $(x, k, h) \in \bar{B}(0, k) \times \mathbb{N} \times B B(S)$,

$$
\begin{aligned}
& A h(x)=\sup _{y \in D} \operatorname{opt}\{p(x, y), u(x, y) h(a(x, y))\}, \\
& B h(x)=\inf _{y \in D} \operatorname{opt}\{q(x, y), v(x, y) h(b(x, y))\} .
\end{aligned}
$$

It follows from $(\varphi, \psi) \in \Phi_{2}$ and (3.20) that (C9) and (C11) imply (C5) and (C7), respectively. Similar to the proof of Theorem 3.5 , by (C10) we deduce that the mapping $H$ maps $B B(S)$ into $B B(S)$ and satisfies that

$$
d_{k}(H h, H g) \leq d_{k}(h, g), \quad \text { for all }(k, h, g) \in \mathbb{N} \times B B(S) \times B B(S),
$$

which means that, for all $(h, g) \in B B(S) \times B B(S)$,

$$
d(H h, H g)=\sum_{k=1}^{\infty} \frac{1}{2^{k}} \cdot \frac{d_{k}(H h, H g)}{1+d_{k}(H h, H g)} \leq \sum_{k=1}^{\infty} \frac{1}{2^{k}} \cdot \frac{d_{k}(h, g)}{1+d_{k}(h, g)}=d(h, g),
$$

that is, the mapping $H$ is nonexpansive in $B B(S)$. Now we show that for each $n \in \mathbb{N}_{0}$

$$
\left|w_{n}(x)\right| \leq \sum_{j=0}^{n} \psi\left(\varphi^{j}(\|x\|)\right), \quad \text { for all }(x, k) \in \bar{B}(0, k) \times \mathbb{N} .
$$

It is clear that (3.24) holds for $n=0$. Assume that (3.24) holds for some $n \in \mathbb{N}_{0}$. In view of $(\mathrm{C} 9),(\mathrm{C} 11),(\mathrm{C} 12),(3.19),(\varphi, \psi) \in \Phi_{2}$ and Lemma 2.1, we derive that

$$
\begin{aligned}
\left|w_{n+1}(x)\right|= & \mid(1-\beta) w_{n}(c(x))+\beta\left(\lambda \sup _{y \in D} \operatorname{opt}\left\{p(x, y), u(x, y) w_{n}(a(x, y))\right\}\right. \\
& \left.+(1-\lambda) \inf _{y \in D} \operatorname{opt}\left\{q(x, y), v(x, y) w_{n}(b(x, y))\right\}\right) \mid \\
\leq & (1-\beta)\left|w_{n}(c(x))\right|+\beta\left(\lambda \sup _{y \in D}\left|\operatorname{opt}\left\{p(x, y), u(x, y) w_{n}(a(x, y))\right\}\right|\right. \\
& \left.+(1-\lambda) \sup _{y \in D}\left|\operatorname{opt}\left\{q(x, y), v(x, y) w_{n}(b(x, y))\right\}\right|\right)
\end{aligned}
$$




$$
\begin{aligned}
\leq & (1-\beta) \sum_{j=0}^{n} \psi\left(\varphi^{j}(\|c(x)\|)\right) \\
& +\beta\left(\lambda \sup _{y \in D} \max \left\{|p(x, y)|,\left|u(x, y) \| w_{n}(a(x, y))\right|\right\}\right. \\
& \left.+(1-\lambda) \sup _{y \in D} \max \left\{|q(x, y)|,\left|v(x, y) \| w_{n}(b(x, y))\right|\right\}\right) \\
\leq & (1-\beta) \sum_{j=0}^{n} \psi\left(\varphi^{j+1}(\|x\|)\right) \\
& +\beta\left(\lambda \sup _{y \in D} \max \left\{\psi(\|x\|), \sum_{j=0}^{n} \psi\left(\varphi^{j}(\|a(x, y)\|)\right)\right\}\right. \\
& \left.+(1-\lambda) \sup _{y \in D} \max \left\{\psi(\|x\|), \sum_{j=0}^{n} \psi\left(\varphi^{j}(\|b(x, y)\|)\right)\right\}\right) \\
\leq & (1-\beta) \sum_{j=0}^{n} \psi\left(\varphi^{j+1}(\|x\|)\right)+\beta\left(\lambda \max \left\{\psi(\|x\|), \sum_{j=0}^{n} \psi\left(\varphi^{j+1}(\|x\|)\right)\right\}\right. \\
& \left.+(1-\lambda) \max \left\{\psi(\|x\|), \sum_{j=0}^{n} \psi\left(\varphi^{j+1}(\|x\|)\right)\right\}\right) \\
\leq & (1-\beta) \sum_{j=0}^{n} \psi\left(\varphi^{j+1}(\|x\|)\right) \\
& +\beta\left(\lambda \sum_{j=0}^{n+1} \psi\left(\varphi^{j}(\|x\|)\right)+(1-\lambda) \sum_{j=0}^{n+1} \psi\left(\varphi^{j}(\|x\|)\right)\right) \leq \sum_{j=0}^{n+1} \psi\left(\varphi^{j}(\|x\|)\right),
\end{aligned}
$$

for all $(x, k, n) \in \bar{B}(0, k) \times \mathbb{N} \times \mathbb{N}_{0}$, that is, (3.24) holds for $n+1$. Consequently (3.24) holds for each $n \in \mathbb{N}_{0}$.

Let $\varepsilon>0, k, n, p \in \mathbb{N}$ and $x_{0} \in \bar{B}(0, k)$. It follows from (3.22) that there exist $y, y_{0}, z, z_{0} \in D$ with

$$
\begin{aligned}
A w_{n+p-1}\left(x_{0}\right)-2^{-1} \varepsilon & <\operatorname{opt}\left\{p\left(x_{0}, y\right), u\left(x_{0}, y\right) w_{n+p-1}\left(a\left(x_{0}, y\right)\right)\right\}, \\
A w_{n-1}\left(x_{0}\right)-2^{-1} \varepsilon & <\operatorname{opt}\left\{p\left(x_{0}, y_{0}\right), u\left(x_{0}, y_{0}\right) w_{n-1}\left(a\left(x_{0}, y_{0}\right)\right)\right\}, \\
A w_{n+p-1}\left(x_{0}\right) & \geq \operatorname{opt}\left\{p\left(x_{0}, y_{0}\right), u\left(x_{0}, y_{0}\right) w_{n+p-1}\left(a\left(x_{0}, y_{0}\right)\right)\right\}, \\
A w_{n-1}\left(x_{0}\right) & \geq \operatorname{opt}\left\{p\left(x_{0}, y\right), u\left(x_{0}, y\right) w_{n-1}\left(a\left(x_{0}, y\right)\right)\right\}
\end{aligned}
$$

and

$$
\begin{aligned}
B w_{n+p-1}\left(x_{0}\right)+2^{-1} \varepsilon & >\operatorname{opt}\left\{q\left(x_{0}, z\right), v\left(x_{0}, z\right) w_{n+p-1}\left(b\left(x_{0}, z\right)\right)\right\}, \\
B w_{n-1}\left(x_{0}\right)+2^{-1} \varepsilon & >\operatorname{opt}\left\{q\left(x_{0}, z_{0}\right), v\left(x_{0}, z_{0}\right) w_{n-1}\left(b\left(x_{0}, z_{0}\right)\right)\right\}, \\
B w_{n+p-1}\left(x_{0}\right) & \leq \operatorname{opt}\left\{q\left(x_{0}, z_{0}\right), v\left(x_{0}, z_{0}\right) w_{n+p-1}\left(b\left(x_{0}, z_{0}\right)\right)\right\}, \\
B w_{n-1}\left(x_{0}\right) & \leq \operatorname{opt}\left\{q\left(x_{0}, z\right), v\left(x_{0}, z\right) w_{n-1}\left(b\left(x_{0}, z\right)\right)\right\} .
\end{aligned}
$$


Combining (C10), (3.25) and (3.26) and using Lemma 2.2, we gain that

$$
\begin{aligned}
A w_{n+p-1}\left(x_{0}\right) & -A w_{n-1}\left(x_{0}\right) \\
< & \operatorname{opt}\left\{p\left(x_{0}, y\right), u\left(x_{0}, y\right) w_{n+p-1}\left(a\left(x_{0}, y\right)\right)\right\} \\
& -\operatorname{opt}\left\{p\left(x_{0}, y\right), u\left(x_{0}, y\right) w_{n-1}\left(a\left(x_{0}, y\right)\right)\right\}+2^{-1} \varepsilon \\
\leq & \left|u\left(x_{0}, y\right)\right|\left|w_{n+p-1}\left(a\left(x_{0}, y\right)\right)-w_{n-1}\left(a\left(x_{0}, y\right)\right)\right|+2^{-1} \varepsilon \\
\leq & \left|w_{n+p-1}\left(a\left(x_{0}, y\right)\right)-w_{n-1}\left(a\left(x_{0}, y\right)\right)\right|+2^{-1} \varepsilon ; \\
A w_{n+p-1}\left(x_{0}\right)- & A w_{n-1}\left(x_{0}\right) \\
> & \operatorname{opt}\left\{p\left(x_{0}, y_{0}\right), u\left(x_{0}, y_{0}\right) w_{n+p-1}\left(a\left(x_{0}, y_{0}\right)\right)\right\} \\
& -\operatorname{opt}\left\{p\left(x_{0}, y_{0}\right), u\left(x_{0}, y_{0}\right) w_{n-1}\left(a\left(x_{0}, y_{0}\right)\right)\right\}-2^{-1} \varepsilon \\
\geq- & \left|u\left(x_{0}, y_{0}\right)\right|\left|w_{n+p-1}\left(a\left(x_{0}, y_{0}\right)\right)-w_{n-1}\left(a\left(x_{0}, y_{0}\right)\right)\right|-2^{-1} \varepsilon \\
\geq- & \left|w_{n+p-1}\left(a\left(x_{0}, y_{0}\right)\right)-w_{n-1}\left(a\left(x_{0}, y_{0}\right)\right)\right|-2^{-1} \varepsilon ; \\
B w_{n+p-1}\left(x_{0}\right) & -B w_{n-1}\left(x_{0}\right) \\
> & \operatorname{opt}\left\{q\left(x_{0}, z\right), v\left(x_{0}, z\right) w_{n+p-1}\left(b\left(x_{0}, z\right)\right)\right\} \\
& -\operatorname{opt}\left\{q\left(x_{0}, z\right), v\left(x_{0}, z\right) w_{n-1}\left(b\left(x_{0}, z\right)\right)\right\}-2^{-1} \varepsilon \\
\geq & -\left|v\left(x_{0}, z\right)\right|\left|w_{n+p-1}\left(b\left(x_{0}, z\right)\right)-w_{n-1}\left(b\left(x_{0}, z\right)\right)\right|-2^{-1} \varepsilon \\
\geq & -\left|w_{n+p-1}\left(b\left(x_{0}, z\right)\right)-w_{n-1}\left(b\left(x_{0}, z\right)\right)\right|-2^{-1} \varepsilon
\end{aligned}
$$

and

$$
\begin{aligned}
B w_{n+p-1}\left(x_{0}\right) & -B w_{n-1}\left(x_{0}\right) \\
< & \operatorname{opt}\left\{q\left(x_{0}, z_{0}\right), v\left(x_{0}, z_{0}\right) w_{n+p-1}\left(b\left(x_{0}, z_{0}\right)\right)\right\} \\
& -\operatorname{opt}\left\{q\left(x_{0}, z_{0}\right), v\left(x_{0}, z_{0}\right) w_{n-1}\left(b\left(x_{0}, z_{0}\right)\right)\right\}+2^{-1} \varepsilon \\
\leq & \left|v\left(x_{0}, z_{0}\right)\right|\left|w_{n+p-1}\left(b\left(x_{0}, z_{0}\right)\right)-w_{n-1}\left(b\left(x_{0}, z_{0}\right)\right)\right|+2^{-1} \varepsilon \\
\leq & \left|w_{n+p-1}\left(b\left(x_{0}, z_{0}\right)\right)-w_{n-1}\left(b\left(x_{0}, z_{0}\right)\right)\right|+2^{-1} \varepsilon,
\end{aligned}
$$

which imply that

$$
\begin{gathered}
\text { (3.27) }\left|A w_{n+p-1}\left(x_{0}\right)-A w_{n-1}\left(x_{0}\right)\right| \\
<\max \left\{\left|w_{n+p-1}\left(a\left(x_{0}, y\right)\right)-w_{n-1}\left(a\left(x_{0}, y\right)\right)\right|,\right. \\
\left.\left|w_{n+p-1}\left(a\left(x_{0}, y_{0}\right)\right)-w_{n-1}\left(a\left(x_{0}, y_{0}\right)\right)\right|\right\}+2^{-1} \varepsilon, \\
(3.28) \quad\left|B w_{n+p-1}\left(x_{0}\right)-B w_{n-1}\left(x_{0}\right)\right| \\
<\max \left\{\left|w_{n+p-1}\left(b\left(x_{0}, z\right)\right)-w_{n-1}\left(b\left(x_{0}, z\right)\right)\right|,\right. \\
\left.\left|w_{n+p-1}\left(b\left(x_{0}, z_{0}\right)\right)-w_{n-1}\left(b\left(x_{0}, z_{0}\right)\right)\right|\right\}+2^{-1} \varepsilon .
\end{gathered}
$$


In light of (3.19), (3.21), (3.22), (3.27) and (3.28), we infer that

$$
\begin{aligned}
\mid w_{n+p}\left(x_{0}\right)- & w_{n}\left(x_{0}\right)|=|(1-\beta) w_{n+p-1}\left(c\left(x_{0}\right)\right)+\beta\left(\lambda A w_{n+p-1}\left(x_{0}\right)\right. \\
+ & \left.(1-\lambda) B w_{n+p-1}\left(x_{0}\right)\right)-(1-\beta) w_{n-1}\left(c\left(x_{0}\right)\right) \\
- & \beta\left(\lambda A w_{n-1}\left(x_{0}\right)+(1-\lambda) B w_{n-1}\left(x_{0}\right)\right) \mid \\
\leq & (1-\beta)\left|w_{n+p-1}\left(c\left(x_{0}\right)\right)-w_{n-1}\left(c\left(x_{0}\right)\right)\right| \\
+ & \beta\left(\lambda\left|A w_{n+p-1}\left(x_{0}\right)-A w_{n-1}\left(x_{0}\right)\right|\right. \\
+ & \left.(1-\lambda)\left|B w_{n+p-1}\left(x_{0}\right)-B w_{n-1}\left(x_{0}\right)\right|\right) \\
< & (1-\beta)\left|w_{n+p-1}\left(c\left(x_{0}\right)\right)-w_{n-1}\left(c\left(x_{0}\right)\right)\right| \\
+ & \beta\left(\lambda \operatorname { m a x } \left\{\left|w_{n+p-1}\left(a\left(x_{0}, y\right)\right)-w_{n-1}\left(a\left(x_{0}, y\right)\right)\right|,\right.\right. \\
& \left.\left|w_{n+p-1}\left(a\left(x_{0}, y_{0}\right)\right)-w_{n-1}\left(a\left(x_{0}, y_{0}\right)\right)\right|\right\}+2^{-1} \lambda \varepsilon \\
+ & (1-\lambda) \max \left\{\left|w_{n+p-1}\left(b\left(x_{0}, z\right)\right)-w_{n-1}\left(b\left(x_{0}, z\right)\right)\right|,\right. \\
& \left.\left.\left|w_{n+p-1}\left(b\left(x_{0}, z_{0}\right)\right)-w_{n-1}\left(b\left(x_{0}, z_{0}\right)\right)\right|\right\}+2^{-1}(1-\lambda) \varepsilon\right) \\
\leq & \max \left\{\left|w_{n+p-1}\left(c\left(x_{0}\right)\right)-w_{n-1}\left(c\left(x_{0}\right)\right)\right|,\right. \\
& \left|w_{n+p-1}\left(a\left(x_{0}, y\right)\right)-w_{n-1}\left(a\left(x_{0}, y\right)\right)\right|, \\
& \left|w_{n+p-1}\left(a\left(x_{0}, y_{0}\right)\right)-w_{n-1}\left(a\left(x_{0}, y_{0}\right)\right)\right|, \\
& \left|w_{n+p-1}\left(b\left(x_{0}, z\right)\right)-w_{n-1}\left(b\left(x_{0}, z\right)\right)\right|, \\
& \left.\left|w_{n+p-1}\left(b\left(x_{0}, z_{0}\right)\right)-w_{n-1}\left(b\left(x_{0}, z_{0}\right)\right)\right|\right\}+2^{-1} \varepsilon \\
= & \left|w_{n+p-1}\left(x_{1}\right)-w_{n-1}\left(x_{1}\right)\right|+2^{-1} \varepsilon
\end{aligned}
$$

for some $y_{1} \in\left\{y, y_{0}, z, z_{0}\right\}$ and $x_{1} \in\left\{c\left(x_{0}\right), a\left(x_{0}, y_{1}\right), b\left(x_{0}, y_{1}\right)\right\}$. Similarly, we get that for each $i \in\{1, \ldots, n\}$ there exist $y_{i} \in D$ and $x_{i} \in\left\{c\left(x_{i-1}\right), a\left(x_{i-1}, y_{i}\right)\right.$, $\left.b\left(x_{i-1}, y_{i}\right)\right\}$ satisfying

$$
\begin{aligned}
& \left|w_{n+p-1}\left(x_{1}\right)-w_{n-1}\left(x_{1}\right)\right|<\left|w_{n+p-2}\left(x_{2}\right)-w_{n-2}\left(x_{2}\right)\right|+2^{-2} \varepsilon, \\
& \left|w_{n+p-2}\left(x_{2}\right)-w_{n-2}\left(x_{2}\right)\right|<\left|w_{n+p-3}\left(x_{3}\right)-w_{n-3}\left(x_{3}\right)\right|+2^{-3} \varepsilon, \\
& \left|w_{p+1}\left(x_{n-1}\right)-w_{1}\left(x_{n-1}\right)\right|<\left|w_{p}\left(x_{n}\right)-w_{0}\left(x_{n}\right)\right|+2^{-n} \varepsilon .
\end{aligned}
$$

Next we show that $\left\{w_{n}\right\}_{n \in \mathbb{N}_{0}}$ is a Cauchy sequence in $(B B(S), d)$. According to $(\varphi, \psi) \in \Phi_{2},(\mathrm{C} 10),(3.24),(3.29)$ and (3.30), we gain that

$$
\begin{aligned}
\left|w_{n+p}\left(x_{0}\right)-w_{n}\left(x_{0}\right)\right| & <\left|w_{p}\left(x_{n}\right)-w_{0}\left(x_{n}\right)\right|+\varepsilon \leq \sum_{j=0}^{p} \psi\left(\varphi^{j}\left(\left\|x_{n}\right\|\right)\right)+\psi\left(\left\|x_{n}\right\|\right)+\varepsilon \\
& \leq \sum_{j=0}^{p} \psi\left(\varphi^{j+n}(k)\right)+\psi\left(\varphi^{n}(k)\right)+\varepsilon \leq \sum_{j=n-1}^{p+n} \psi\left(\varphi^{j}(k)\right)+\varepsilon,
\end{aligned}
$$


which means that $d_{k}\left(w_{n+p}, w_{n}\right) \leq \sum_{j=n-1}^{n+p} \psi\left(\varphi^{j}(k)\right)+\varepsilon$, taking $\varepsilon \rightarrow 0^{+}$in the above inequality, we infer that

$$
d_{k}\left(w_{n+p}, w_{n}\right) \leq \sum_{j=n-1}^{n+p} \psi\left(\varphi^{j}(k)\right) .
$$

Observe that $\sum_{n=0}^{\infty} \psi\left(\varphi^{n}(t)\right)<+\infty$ for each $t>0$. Thus (3.31) guarantees that $\left\{w_{n}\right\}_{n \in \mathbb{N}_{0}}$ is a Cauchy sequence in $(B B(S), d)$ and it converges to some $w \in$ $B B(S)$. Letting $p \rightarrow \infty$ in (3.31), we know immediately that

$$
d_{k}\left(w_{n}, w\right) \leq \sum_{j=n-1}^{\infty} \psi\left(\varphi^{j}(k)\right), \quad \text { for all }(k, n) \in \mathbb{N} \times \mathbb{N} .
$$

In terms of (3.23), we see that

$d(H w, w) \leq d\left(H w, H w_{n}\right)+d\left(w_{n+1}, w\right) \leq d\left(w, w_{n}\right)+d\left(w_{n+1}, w\right) \rightarrow 0$ as $n \rightarrow \infty$, which yields that $H w=w$, that is, the functional equation (1.5) possesses a solution $w \in B B(S)$.

Next we show $(\mathrm{C} 13)$. Let $(x, k) \in \bar{B}(0, k) \times \mathbb{N}$. Using (C10), (3.24) and $(\varphi, \psi) \in \Phi_{2}$, we conclude that

$|w(x)| \leq\left|w(x)-w_{n}(x)\right|+\left|w_{n}(x)\right| \leq d_{k}\left(w, w_{n}\right)+\sum_{j=0}^{n} \psi\left(\varphi^{j}(\|x\|)\right) \rightarrow \sum_{j=0}^{\infty} \psi\left(\varphi^{j}(\|x\|)\right)$ as $n \rightarrow \infty$, that is, (C13) holds.

Next we show (C14). Given $\left(x_{0}, k\right) \in \bar{B}(0, k) \times \mathbb{N}$ and $\left\{y_{n}\right\}_{n \in \mathbb{N}} \subset D$ with $x_{n} \in\left\{c\left(x_{n-1}\right), a\left(x_{n-1}, y_{n}\right), b\left(x_{n-1}, y_{n}\right)\right\}$ for each $n \in \mathbb{N}$. It follows from (C11), (3.20) and $(\varphi, \psi) \in \Phi_{2}$ that

$$
\begin{aligned}
\left\|x_{n}\right\| & \leq \max \left\{\left\|a\left(x_{n-1}, y_{n}\right)\right\|,\left\|b\left(x_{n-1}, y_{n}\right)\right\|,\left\|c\left(x_{n-1}\right)\right\|\right\} \\
& \leq \varphi\left(\left\|x_{n-1}\right\|\right) \leq \ldots \leq \varphi^{n}\left(\left\|x_{0}\right\|\right) \leq \varphi^{n}(k)<k,
\end{aligned}
$$

for all $n \in \mathbb{N}$, which together with (3.24) implies that

$$
\begin{aligned}
\left|w\left(x_{n}\right)\right| & \leq\left|w\left(x_{n}\right)-w_{n}\left(x_{n}\right)\right|+\left|w_{n}\left(x_{n}\right)\right| \\
& \leq d_{k}\left(w, w_{n}\right)+\sum_{j=0}^{n} \psi\left(\varphi^{j}\left(\left\|x_{n}\right\|\right)\right) \leq d_{k}\left(w, w_{n}\right)+\sum_{j=n}^{2 n} \psi\left(\varphi^{j}(k)\right) \rightarrow 0
\end{aligned}
$$

as $n \rightarrow \infty$, which yields that $\lim _{n \rightarrow \infty} w\left(x_{n}\right)=0$.

Finally, we show (C15). Suppose that the functional equation (1.5) has another solution $h \in B B(S)$ that satisfies (C14). 
Given $\left(x_{0}, k\right) \in \bar{B}(0, k) \times \mathbb{N}$ and $\varepsilon>0$. It follows from (3.22) that there exist $y, y_{0}, z, z_{0} \in D$ with

$$
\begin{aligned}
A w\left(x_{0}\right)-2^{-1} \varepsilon & <\operatorname{opt}\left\{p\left(x_{0}, y\right), u\left(x_{0}, y\right) w\left(a\left(x_{0}, y\right)\right)\right\}, \\
A h\left(x_{0}\right)-2^{-1} \varepsilon & <\operatorname{opt}\left\{p\left(x_{0}, y_{0}\right), u\left(x_{0}, y_{0}\right) h\left(a\left(x_{0}, y_{0}\right)\right)\right\}, \\
A w\left(x_{0}\right) & \geq \operatorname{opt}\left\{p\left(x_{0}, y_{0}\right), u\left(x_{0}, y_{0}\right) w\left(a\left(x_{0}, y_{0}\right)\right)\right\}, \\
A h\left(x_{0}\right) & \geq \operatorname{opt}\left\{p\left(x_{0}, y\right), u\left(x_{0}, y\right) h\left(a\left(x_{0}, y\right)\right)\right\}, \\
B w\left(x_{0}\right)+2^{-1} \varepsilon & >\operatorname{opt}\left\{q\left(x_{0}, z\right), v\left(x_{0}, z\right) w_{n+p-1}\left(b\left(x_{0}, z\right)\right)\right\}, \\
B h\left(x_{0}\right)+2^{-1} \varepsilon & >\operatorname{opt}\left\{q\left(x_{0}, z_{0}\right), v\left(x_{0}, z_{0}\right) h\left(b\left(x_{0}, z_{0}\right)\right)\right\}, \\
B w\left(x_{0}\right) & \leq \operatorname{opt}\left\{q\left(x_{0}, z_{0}\right), v\left(x_{0}, z_{0}\right) w_{n+p-1}\left(b\left(x_{0}, z_{0}\right)\right)\right\}, \\
B h\left(x_{0}\right) & \leq \operatorname{opt}\left\{q\left(x_{0}, z\right), v\left(x_{0}, z\right) h\left(b\left(x_{0}, z\right)\right)\right\},
\end{aligned}
$$

which together with (C10), (3.21) and (3.22) yield that

$$
\begin{aligned}
\mid w\left(x_{0}\right)- & h\left(x_{0}\right)|=| \lambda A w\left(x_{0}\right)+(1-\lambda) B w\left(x_{0}\right)-\lambda A h\left(x_{0}\right)-(1-\lambda) B h\left(x_{0}\right) \mid \\
\leq & \lambda\left|A w\left(x_{0}\right)-A h\left(x_{0}\right)\right|+(1-\lambda)\left|B w\left(x_{0}\right)-B h\left(x_{0}\right)\right| \\
< & \lambda \max \left\{\left|u\left(x_{0}, y\right)\right|\left|w\left(a\left(x_{0}, y\right)\right)-h\left(a\left(x_{0}, y\right)\right)\right|,\right. \\
& \left.\left|u\left(x_{0}, y_{0}\right)\right|\left|w\left(a\left(x_{0}, y_{0}\right)\right)-h\left(a\left(x_{0}, y_{0}\right)\right)\right|\right\}+2^{-1} \lambda \varepsilon \\
+ & (1-\lambda) \max \left\{\left|v\left(x_{0}, z\right)\right|\left|w\left(b\left(x_{0}, z\right)\right)-h\left(b\left(x_{0}, z\right)\right)\right|,\right. \\
& \left.\left|v\left(x_{0}, z_{0}\right)\right|\left|w\left(b\left(x_{0}, z_{0}\right)\right)-h\left(b\left(x_{0}, z_{0}\right)\right)\right|\right\}+2^{-1}(1-\lambda) \varepsilon \\
\leq & \max \left\{\left|w\left(a\left(x_{0}, y\right)\right)-h\left(a\left(x_{0}, y\right)\right)\right|,\left|w\left(a\left(x_{0}, y_{0}\right)\right)-h\left(a\left(x_{0}, y_{0}\right)\right)\right|\right. \\
& \left.\left|w\left(b\left(x_{0}, z\right)\right)-h\left(b\left(x_{0}, z\right)\right)\right|,\left|w\left(b\left(x_{0}, z_{0}\right)\right)-h\left(b\left(x_{0}, z_{0}\right)\right)\right|\right\}+2^{-1} \varepsilon \\
= & \left|w\left(x_{1}\right)-h\left(x_{1}\right)\right|+2^{-1} \varepsilon
\end{aligned}
$$

for some $x_{1} \in\left\{a\left(x_{0}, y_{1}\right), b\left(x_{0}, y_{1}\right)\right\}$ and $y_{1} \in\left\{y, y_{0}, z, z_{0}\right\}$, that is,

$$
\left|w\left(x_{0}\right)-h\left(x_{0}\right)\right| \leq\left|w\left(x_{1}\right)-h\left(x_{1}\right)\right|+2^{-1} \varepsilon .
$$

Proceeding in this way, we get that for each $n \in \mathbb{N} \backslash\{1\}$, there exist $x_{i} \in$ $\left\{a\left(x_{i-1}, y_{i}\right), b\left(x_{i-1}, y_{i}\right)\right\}$ and $y_{i} \in D, i \in\{2, \ldots, n\}$, satisfying

$$
\begin{aligned}
&\left|w\left(x_{1}\right)-h\left(x_{1}\right)\right| \leq\left|w\left(x_{2}\right)-h\left(x_{2}\right)\right|+2^{-2} \varepsilon, \\
&\left|w\left(x_{2}\right)-h\left(x_{2}\right)\right| \leq\left|w\left(x_{3}\right)-h\left(x_{3}\right)\right|+2^{-3} \varepsilon, \\
& \ldots \ldots \ldots \ldots \ldots \ldots \ldots \ldots \ldots \ldots \ldots \ldots \ldots \ldots \ldots \\
&\left|w\left(x_{n-1}\right)-h\left(x_{n-1}\right)\right| \leq\left|w\left(x_{n}\right)-h\left(x_{n}\right)\right|+2^{-n} \varepsilon .
\end{aligned}
$$

Using (3.32) and (3.33), we infer that $\left|w\left(x_{0}\right)-h\left(x_{0}\right)\right| \leq\left|w\left(x_{n}\right)-h\left(x_{n}\right)\right|+\varepsilon \rightarrow \varepsilon$ as $n \rightarrow \infty$, letting $\varepsilon \rightarrow 0^{+}$in the above inequality, we derive that $w\left(x_{0}\right)=h\left(x_{0}\right)$.

REMARK 3.9. Theorem 3.8 generalizes and unifies Theorems 3.4 and 3.5 in [4], Theorem 3.5 in [6], Corollaries 2.2 and 2.3 in [8], Theorem 3.5 in [9], 
Corollaries 3.1, 3.3 and 3.4 in [11], Theorems 2.3 and 2.4 in [12], Theorem 3.4 in [15]. The example below shows that Theorem 3.8 extends properly the corresponding results in [4], [6], [8], [9], [11], [12] and [15].

ExAmPle 3.10. Consider the functional equation

$$
\begin{aligned}
& f(x)=\lambda \sup _{y \in \mathbb{R}} \text { opt }\left\{\frac{x^{9} y^{2} \sin \left(x^{8}-y^{6}\right)}{x^{2} y^{2}+1}, \sin ^{3}\left(\sqrt{x} y^{2}\right) f\left(\frac{x \ln \left(1+\frac{1}{1+x^{2} y^{6}}\right)}{4+\cos ^{9}\left(x^{5} y^{7}\right)}\right)\right\} \\
& +(1-\lambda) \inf _{y \in \mathbb{R}} \text { opt }\left\{\frac{x^{8} \arctan ^{7}\left(x^{5} y^{3}\right)}{x+y^{2}+1}, \cos ^{5}\left(x^{9} y^{4}\right) f\left(\frac{x^{6} \sin ^{2}\left(x^{2} y^{4}\right)}{3 x^{5}+y^{8}+1}\right)\right\},
\end{aligned}
$$

for all $x \in \mathbb{R}^{+}$. Let $\lambda \in[0,1], \beta \in(0,1], X=Y=\mathbb{R}, S=\mathbb{R}^{+}, D=\mathbb{R}$. Let $p, q, u, v: S \times D \rightarrow \mathbb{R}, a, b: S \times D \rightarrow S, c: S \rightarrow S$ and $\varphi, \psi: \mathbb{R}^{+} \rightarrow \mathbb{R}^{+}$be defined by

$$
\begin{aligned}
p(x, y) & =\frac{x^{9} y^{2} \sin \left(x^{8}-y^{6}\right)}{x^{2} y^{2}+1}, & q(x, y) & =\frac{x^{8} \arctan ^{7}\left(x^{5} y^{3}\right)}{x+y^{2}+1}, \\
u(x, y) & =\sin ^{3}\left(\sqrt{x} y^{2}\right), & v(x, y) & =\cos ^{5}\left(x^{9} y^{4}\right), \\
a(x, y) & =\frac{x \ln \left(1+\frac{1}{1+x^{2} y^{6}}\right)}{4+\cos ^{9}\left(x^{5} y^{7}\right)}, & b(x, y) & =\frac{x^{6} \sin ^{2}\left(x^{2} y^{4}\right)}{3 x^{5}+y^{8}+1}, \\
c(x) & =\frac{\sin ^{2} \sqrt{x}}{5+2 \cos \left(x^{9}-8 x^{7}+3 x^{6}-x-1\right)}, & & \text { for all }(x, y) \in S \times D, \\
\varphi(t) & =\frac{t}{3}, \quad \psi(t)=36 t^{7}, & & \text { for all } t \in \mathbb{R}^{+} .
\end{aligned}
$$

For any $w_{0} \in B B(S)$ with $\left|w_{0}(x)\right| \leq \psi(\|x\|)$, for all $(x, k) \in \bar{B}(0, k) \times \mathbb{N}$, define the iterative sequence $\left\{w_{n}\right\}_{n \in \mathbb{N}_{0}}$ by

$$
\begin{aligned}
& w_{n+1}(x)=(1-\beta) w_{n}\left(\frac{\sin ^{2} \sqrt{x}}{5+2 \cos \left(x^{9}-8 x^{7}+3 x^{6}-x-1\right)}\right) \\
& +\beta\left[\lambda \sup _{y \in D} \text { opt }\left\{\frac{x^{9} y^{2} \sin \left(x^{8}-y^{6}\right)}{x^{2} y^{2}+1}, \sin ^{3}\left(\sqrt{x} y^{2}\right) w_{n}\left(\frac{x \ln \left(1+\frac{1}{1+x^{2} y^{6}}\right)}{4+\cos ^{9}\left(x^{5} y^{7}\right)}\right)\right\}\right. \\
& \left.+(1-\lambda) \inf _{y \in D} \text { opt }\left\{\frac{x^{8} \arctan \left(x^{5} y^{3}\right)}{x+y^{2}+1}, \cos ^{5}\left(x^{9} y^{4}\right) w_{n}\left(\frac{x^{6} \sin ^{2}\left(x^{2} y^{4}\right)}{3 x^{5}+y^{8}+1}\right)\right\}\right],
\end{aligned}
$$

for all $(x, k, n) \in \bar{B}(0, k) \times \mathbb{N} \times \mathbb{N}_{0}$. Obviously, the conditions of Theorem 3.4 hold. It follows from Theorem 3.4 that the functional equation (3.34) has a unique solution $w \in B B(S)$ satisfying $(\mathrm{C} 12)-(\mathrm{C} 15)$. In particular, the iterative sequence $\left\{w_{n}\right\}_{n \in \mathbb{N}_{0}}$ converges to $w$. However, Theorems 3.4 and 3.5 in [4], Theorem 3.5 in [6], Corollaries 2.2 and 2.3 in [8], Theorem 3.5 in [9], Corollaries 3.1, 3.3 and 3.4 in [11], Theorems 2.3 and 2.4 in [12], Theorem 3.4 in [15] are not applicable for the functional equation (3.34).

Acknowledgements. The authors would like to thank the referees for useful comments and suggestions. 


\section{REFERENCES}

[1] R. Bellman, Dynamic Programming, Princeton University Press, Princeton, New Jersey, 1957.

[2] R. Bellman and E. S. Lee, Functional equations arising in dynamic programming, Aequationes Math. 17 (1978), 1-18.

[3] R. Bellman and M. Roosta, A technique for the reduction of dimensionality in dynamic programming, J. Math. Anal. Appl. 88 (1982), 543-546.

[4] P.C. Bhakta And S.R. Choudhury, Some existence theorems for functional equations arising in dynamic programming II, J. Math. Anal. Appl. 131 (1988), 217-231.

[5] P.C. Bhakta And S. Mitra, Some existence theorems for functional equations arising in dynamic programming, J. Math. Anal. Appl. 98 (1984), 348-362.

[6] Z. LiU, Existence theorems of solutions for certain classes of functional equations arising in dynamic programming, J. Math. Anal. Appl. 262 (2001), 529-553.

[7] Z. Liu, R.P. Agarwal and S.M. Kang, On solvability of functional equations and system of functional equations arising in dynamic programming, J. Math. Anal. Appl. 297 (2004), 111-130.

[8] Z. LiU And S.M. Kang, Properties of solutions for certain functional equations arising in dynamic programming, J. Global Optim. 34 (2006), 273-292.

[9] Existence and uniqueness of solutions for two classes of functional equations arising in dynamic programming, Acta Math. Appl. Sini. 23 (2007), 195-208.

[10] Z. Liu, S.M. KAng AND J.S. UmE, Solvability and convergence of iterative algorithms for certain functional equations arising in dynamic programming, Optimization 59(6) (2010), 887-916.

[11] Z. LiU AND J.S. Ume, On properties of solutions for a class of functional equations arising in dynamic programming, J. Optim. Theory Appl. 117 (2003), 533-551.

[12] Z. Liu, J.S. Ume AND S.M. KANG, Some existence theorems for functional equations arising in dynamic programming, J. Korean Math. Soc. 43 (2006), 11-28.

[13] Some existence theorems for functional equations and system of functional equations arising in dynamic programming, Taiwanese J. Math. 14 (2010), 1517-1536.

[14] Z. Liu, Y.G. Xu, J.S. Ume And S.M. Kang, Solutions to two functional equations arising in dynamic programming, J. Comput. Appl. Math. 192 (2006), 251-269.

[15] Z. Liu, L. Zhao, S.M. Kang and J.S. Ume, On the solvability of a functional equation, Optimization 60 (2011), 365-375.

ZEQING LIU

Department of Mathematics

Liaoning Normal University

Dalian, Liaoning 116029, P.R. CHINA

E-mail address: zeqingliu@163.com

Shin Min KAng

Department of Mathematics and RINS

Gyeongsang National University

Jinju 660-701, KOREA

E-mail address:smkang@gnu.ac.kr

TMNA : Volume $46-2015-\mathrm{N}^{\circ} 1$ 